\title{
Dissociating perception and action in Kanizsa's compression illusion
}

\author{
NICOLA BRUNO and PAOLO BERNARDIS \\ Università di Trieste, Trieste, Italy
}

\begin{abstract}
When a horizontally elongated surface is occluded in the middle by a larger surface, it appears narrower than its true width (Kanizsa's compression illusion). We report that a similar compression effect occurs for closed-loop visuomotor matches of size, but not for otherwise comparable open-loop "mimed" reaching or size-matching visuomotor responses. Our study is the first in which a comparison of size perception in personal space with bilateral actions performed with both hands (instead of precision grips employing the thumb and the index finger) is used to investigate motor responses to Kanizsa's compression illusion. Implications for the current debate on the existence of dissociations between spatial perception and visually controlled actions in personal space are discussed.
\end{abstract}

Over the last decade, functional dissociations between perception and action in neurologically intact individuals have been subjected to increased scrutiny in vision science. Such dissociations in healthy individuals are commonly interpreted as evidence for a functional difference between two processing streams originating at the primary visual area (V1) of the primate brain. According to a widely held view (Milner \& Goodale, 1995), the ventral projection from V1 to the inferotemporal (IT) cortex processes visual information to construct conscious representations of distal objects and events, whereas the dorsal projection from V1 to the posterior parietal (PP) cortex processes visual information to control action, independently of visual phenomenology (see also Bridgeman, Kirch, \& Sperling, 1981). This characterization of the functional organization of the visual system has been called the two-visual-systems hypothesis, or TVSH (DeLucia, Tresilian, \& Meyer, 2000).

To a vision scientist, the TVSH is theoretically attractive for several reasons. First of all, the TVSH may provide a theoretical means by which to reconcile ecological and constructivist theories of perception (Bruce, Green, \& Georgeson, 1996). In addition, the TVSH provides an elegant explanation for dissociations observed in neuropsychological patients with PP and IT lesions (Milner et al., 1991; Perenin $\&$ Vighetto, 1983). Most critical to the issues investigated in the present paper, finally, the TVSH may explain a num-

Preliminary versions of this work were presented at the annual Trieste Symposium on Perception and Cognition, Trieste, Italy, October 19-21, 2000 and at the First Annual Meeting of the Vision Sciences Society, Sarasota, FL, May 4-8, 2001. This work was supported in part by MURST Grant 9911333852 to the first author. We thank Marco Detoni for help in collecting the data and Tiziano Agostini for advertising the study to first-year undergraduates. We also thank Peter Vishton, Volker Franz, and an anonymous reviewer for their comments on earlier versions of this report. Correspondence concerning this article should be addressed to N. Bruno, Dipartimento di Psicologia, Università di Trieste, via S. Anastasio, 12, 34134 Trieste, Italy (e-mail: nicola.bruno@ univ.trieste.it). ber of well-documented functional dissociations observed in healthy observers, such as those between visual and motoric responses to surface slope (Bhalla \& Proffitt, 1999; Proffitt, Bhalla, Gossweiler, \& Midgett, 1995), between distance judgments and walking responses (Loomis, Da Silva, Fujita, \& Fukushima, 1992; Wraga, Creem, \& Proffitt, 2000), or between visually perceived motion and ocular tracking and pointing (Bridgeman, 1999; Bridgeman, Lewis, Heit, \& Nagle, 1979).

Functional dissociations between perceptual and motor responses in healthy observers are predicted by one critical feature of the TVSH. According to the TVSH, mental operations carried out by the dorsal stream to plan and control actions are short-lived, fast, and based on observerrelative frames of reference. Conversely, mental operations carried out by the ventral stream involve longer lasting representations that privilege object-relative frames useful for object constancy and recognition. Operations relating an object to its context are known to produce systematic deviations from accuracy in several configurations that produce well-known geometrical illusions. Observercentered locations or distances used to control action need not emphasize such relations and, hence, may not show similar biases. Thus, it is a specific prediction of the TVSH of Milner and Goodale (1995) that one should find spatial tasks that produce illusory biases in conscious perception while still allowing accurate motor responses.

Given the theoretical interest of the TVSH, recent findings on perception-action dissociations are especially worth evaluating. A review of the literature revealed that opposing results have been reported concerning relatively small extents within an observer's "personal" space (see Cutting \& Vishton, 1995) and comparisons of perceptual matches with grasping actions involving the finger and the thumb. For instance, dissociations between perception and grasping have been reported with small-scale surfaces yielding well-known illusions in conscious perception. These included the Ebbinghaus-Titchener circles illusion 


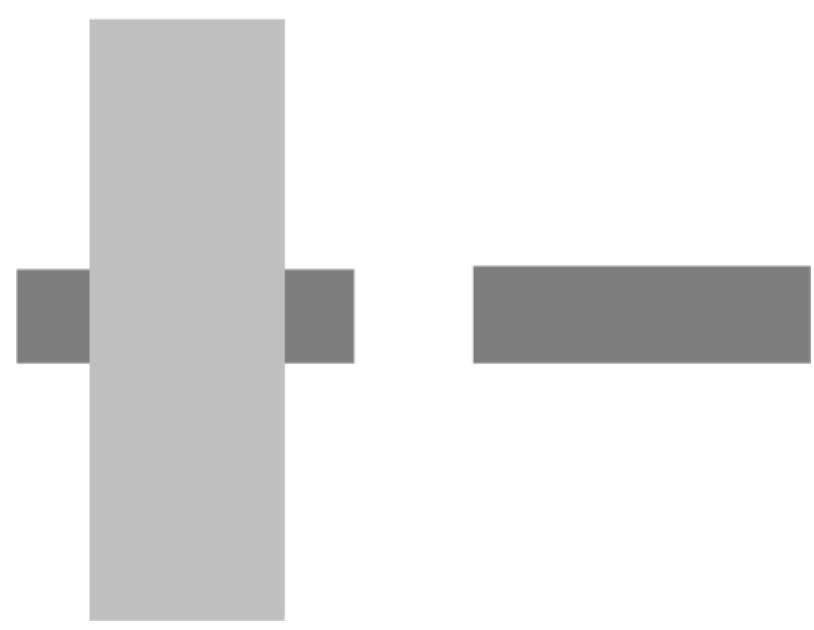

Figure 1. Kanizsa's compression illusion. The dark gray horizontal surfaces have the same width. The left one appears narrower. The compression effect is around $5 \%$ of the actual width in these conditions.

(Aglioti, DeSouza, \& Goodale, 1995; Haffenden \& Goodale, 1998), the vertical-horizontal segments illusion (Vishton \& Cutting, 1995), the Müller-Lyer arrows illusion (Gentilucci, Chieffi, Daprati, Saetti, \& Toni, 1996; Ottode-Haart, Carey, \& Milne, 1999), and the Ponzo diverginglines illusion (Brenner \& Smeets, 1996; Jackson \& Shaw, 2000). In contrast with these findings, however, some studies have reported comparable biases in perception and grasping in the Müller-Lyer arrows (Daprati \& Gentilucci, 1997; Post \& Welch, 1996; Vishton, Cutting, \& Rea, 1997) and in the Ebbinghaus-Titchener circles (V. H. Franz, Gegenfurtner, Bülthoff, \& Fahle, 2000; Pavani, Boscagli, Benvenuti, Rabuffetti, \& Farne, 1999).

Although current opinions about the import of these seeming contradictions differ (Bruno, 2001; Carey, 2001; Plodowsky \& Jackson, 2001), several investigators have noted that the perceptual and the motor tasks must be adequately matched before any meaningful comparison can be made between them. For instance, in the EbbinghausTitchener illusion, a strong size-contrast effect is measured perceptually when observers directly compare two target circles. No such comparison is needed in the action task. In fact, grasping actions are directed to one target at a time. Thus, grasping is not fully comparable with the perceptual task, in that it does not involve a simultaneous appreciation of two sizes relative to their contrasting surrounds. Accordingly, some have reported that when the perceptual task is modified to remove such simultaneous appreciation, the perceptual size-contrast effect reduces and becomes similar to the effect on the grasping response (V. H. Franz et al., 2000; Pavani et al., 1999). It remains to be established whether these findings should be taken as evidence against the TVSH or whether they reflect effects on grasping responses owing to the distance between the target disks and the flankers, rather than the targetflankers size contrast (Haffenden, Schiff, \& Goodale, 2001).
Whatever the interpretation, the above results remain important in that they suggest that the methodology of comparisons between perceptual and motor responses must be evaluated carefully before any claim is made that the two kinds of responses can be dissociated. In a recent study of precision grips in the well-known horizontalvertical illusion, Vishton, Rea, Cutting, and Nunez (1999) carefully matched perceptual and motor responses and showed that the illusion can be produced in both perception and action, depending on conditions. Specifically, Vishton and his collaborators performed four experiments on perceptual and motoric responses to different versions of the vertical-horizontal illusion. In the first experiment, grip scaling showed essentially no underestimation of the vertical, whereas perceptual size estimates did. In the second and third experiments, perceptual estimates also failed to show the underestimation, provided that the observer's attention was directed to a single element of the display. Finally, in a fourth experiment, grip scaling showed a marked underestimation of the horizontal when three fingers were used to reach for the vertices of a triangular figure.

Thus, in the study of Vishton and collaborators, the horizontal-vertical illusion deceived both the eye and the hand, depending on whether the task emphasized objector observer-relative ("absolute") metrics. Similar findings have also been reported in a study of a walkable MüllerLyer configuration (Wraga et al., 2000). Thus, at least two studies in which visual and motoric tasks were matched adequately confirmed that perceptual and motoric distances can show functional dissociations. It is also not clear whether these results can be reconciled with the TVSH as proposed by Milner and Goodale (1995). Insofar as they demonstrated effects of both object- and observer-relative frames of reference in both perceptual and action tasks, these studies seem to imply that the original characterization of the two visual streams is, at best, too rigid. Given these concerns and the somewhat mixed evidence concerning potential dissociations between perceptual judgments and motoric responses performed in personal space, it seems important to continue examining perceptual and motoric performance in these conditions.

In this paper, we report a study of the visual planning of actions on amodally completed objects presented within an observer's reach. In conscious visual perception, an elongated surface appears compressed relative to its true width when presented behind an occluder (Kanizsa, 1972; see Figure 1). Several explanations have been offered for the compression illusion, including representational economy for amodally completed surface parts (Kanizsa's own favored explanation), size contrast, and others (for reviews, see Luccio, 1983, and Vezzani, 1999). Whatever the explanation, the illusory compression has been observed in a variety of conditions, yielding illusory compressions on the order of 4\%-6\% (e.g., Gerbino, 1979; Kanizsa, 1975; Tampieri, 1979; Zanuttini, 1977). We sought to determine whether a similar compression effect would affect visually planned actions in personal space and how. To this aim, we performed two experiments. In the first, we showed that bilateral actions such as those investigated here are 
normally well scaled to an object's width. In the second, we compared these actions with perceptual discriminative responses in order to test for a potential dissociation.

\section{EXPERIMENT 1}

To evaluate the motor responses investigated in our study, in a preliminary experiment, we investigated how well two-hand separations reflect an object's size when an observer is requested to mime a grasp of such a surface or when he or she matches with the hands the surface's horizontal extent. Similar experiments involving two-finger precision grips have shown that measures of grip aperture are highly correlated with an object's physical size (e.g., Marteniuk, Leavitt, MacKenzie, \& Athenes, 1990). However, no comparable motor data are presently available for two-hand bilateral actions, which require coordination of the two limbs and may be more difficult to perform than actions involving a single hand (Boessenkool, Nijhof, \& Erkelens, 1999; E. A. Franz, Zelaznik, Swinnen, \& Walter, 2001). Given that two-hand grasps are in fact commonly performed in a number of everyday actions, such as picking up a box or holding a car wheel, the information provided by such a study has general interest. In the context of our study of Kanizsa's compression illusion, however, the focus of our interest is in determining whether open- or closed-loop two-hand gestures can be used as indicators of motor representations of a surface's extent. To this aim, we presented rectangular surfaces of variable horizontal width within an observer's personal space and measured three kinds of two-hand actions.

\section{Method}

\section{Observers}

Four observers participated in this study. One of them (P.B.) was the second author of this paper and was fully aware of the purposes of the present measurements. The other three were naive as to these purposes. All the participants had normal vision or wore prescriptions that were customary for them.

\section{Design and Conditions}

Three kinds of motor responses were investigated: a simultaneous reach with both hands for the vertical sides of a horizontally elongated rectangular surface (open-loop mimed reaching task), a match performed with both hands in front of the actor's chest, with no extension of the arms toward the surface (open-loop matching task), and an intermodal match of the surface's width with the distance between the hands, performed with the surface on the side of the observer rather than in front (closed-loop matching task). The openloop reaching and matching actions were performed while a blindfold was worn. The closed-loop match was performed under visual control, although the target surface and the hands could not be viewed simultaneously. In addition to the task manipulation, the initial position of the actor's hands was also varied. Actors initiated their responses with their hands either raised above their shoulders or joined in front of their chests. Finally, each response in any given condition was replicated two times. This yielded a total of 36 visuomotor responses, which were performed in a completely randomized order by each participant.

\section{Materials}

The stimulus materials consisted of rectangular surfaces cut out of light plasterboard and resembled the patterns presented in Fig- ure 1 . The surfaces were painted dark gray and were $6 \mathrm{~cm}$ vertically and $19.5,23.5$, or $27.5 \mathrm{~cm}$ horizontally. All the surfaces were attached at the end of a metal rod fixed on a stand and were placed at a $0.5-\mathrm{m}$ distance from the actor's viewpoint. Actions were recorded by means of a digital camera (Kodak Dc 240) and were analyzed by importing image files into a personal computer equipped with the Adobe Photoshop image-processing software.

\section{Procedure}

Given that the required responses varied according to conditions, minor procedural differences were present between the three visuomotor conditions. Overall, however, care was taken to ensure that all the conditions yielded comparable measures and variability. The exact measurement procedures were as follows.

Open-loop mimed reach. The observers sat in front of one of the rectangular surfaces having a randomly selected horizontal size. They were told to extend their arms, to aim toward the dark-gray rectangle, and to position their hands next to the left and right vertical sides, as if they wanted to hold it as one would hold a car wheel. To counterbalance across antagonistic muscles and body positions, each measure of the action was an average of two trials in randomized order. In one trial, the action was initiated with the hands joined in front of the chest. In the other trial, it was initiated with the hands raised above the shoulders. To ensure that no visual feedback was available during the action, a mask was used to blindfold the participant after he or she had completed the necessary visual inspection of the target. Thus, the exact sequence of events in this condition was as follows. First, the target stand was positioned in front of the seated observer at the appropriate distance. At this point, the observer assumed one randomly chosen starting position (hands up or to the chest), and the experimenter asked the observer to look at the target carefully to prepare for the action. As soon as the observer was ready, the experimenter lowered the mask over his or her eyes. This event also signaled that the action could begin. The observer then extended his or her arms and positioned his or her hands while the target was removed to avoid actual contact. This ensured that the observer did not obtain tactile feedback about his or her hand position. As soon as the action was completed, a meter was placed horizontally across the hands at the bases of the observer's thumbs, and a photograph was taken by a research assistant (see Figure 2). At this point, the participant was instructed to remove the blindfold. This ensured that he or she could not visually check hand position against the display.

Open-loop match. The procedure was exactly the same as that used in the two-hands reaching condition, except that the observers were told that they had to position their hands so as to match the remembered horizontal extent of the dark gray rectangle with the distance between their hands. Thus, the action isolated the motoric representation of the surfaces's horizontal extent, rather than its position in three-dimensional space.

Closed-loop match. The procedure was similar to that used in the memory match, with the following exceptions. First, to investigate motoric size estimates employing visual feedback, matches were performed without a blindfold. In addition, to prevent attempts to visually align the hands with the sides of the rectangle, the surface was placed on the side of the observer at the usual distance of $57 \mathrm{~cm}$. The observers were required to assume the same starting positions as those used in the other two motor conditions, turn their heads to look at the target surface, and perform one initial match. Once they had their hands in this initial position, they were allowed to look at the surface again in order to modify the match as many times as they deemed necessary.

\section{Analysis}

After each participant had completed the experiment, image files were downloaded to a Macintosh PowerPC computer. Hand separations after completion of each action were measured from these image files, using the Adobe Photoshop image-processing software. 


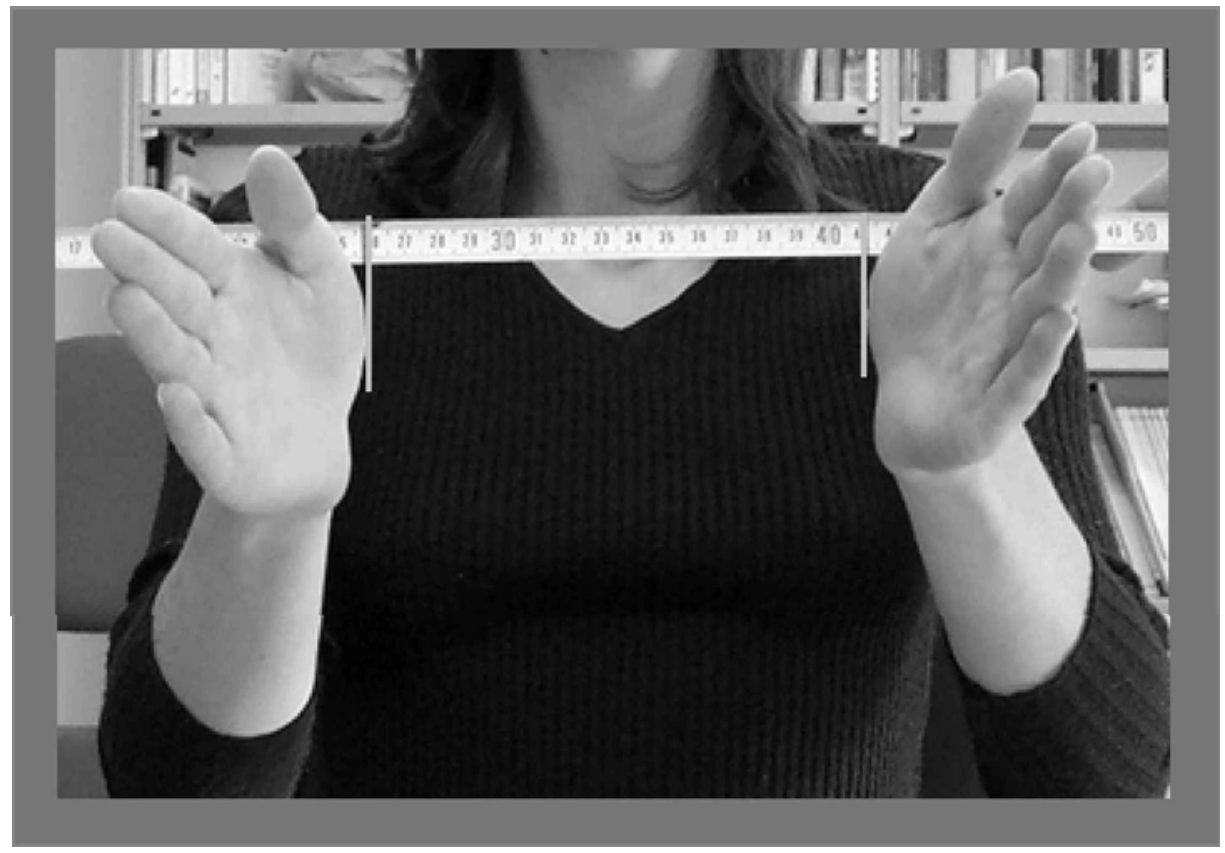

Figure 2. In all motor conditions, hand separations at completion of motor responses were recorded by photographs. Immediately before recording, a meter was placed across the hands in correspondence with the bases of the thumbs. Hand separations after completion of each action were measured from image files by drawing tangents to the bases of the thumbs and extending them until they intersected the photographed meter.

Tangents to the bases of the thumbs were drawn on the pictures and were extended until they intersected the photographed meter. Hand separations were then simply read off the meter.

\section{Results and Discussion}

Hand separation measures in the three visuomotor responses were averaged across repetitions and starting positions and were plotted individually against the actual horizontal extension of the rectangular surface (Figure 3). In all three visuomotor conditions, all the participants showed excellent scaling of hand separation to actual extent. To ensure that these averages did not hide some unknown interaction with hand starting position, we also averaged the data across observers after dividing by starting position. This replot showed no hint of such an interaction. We interpret the above results as evidence that hand sepa-

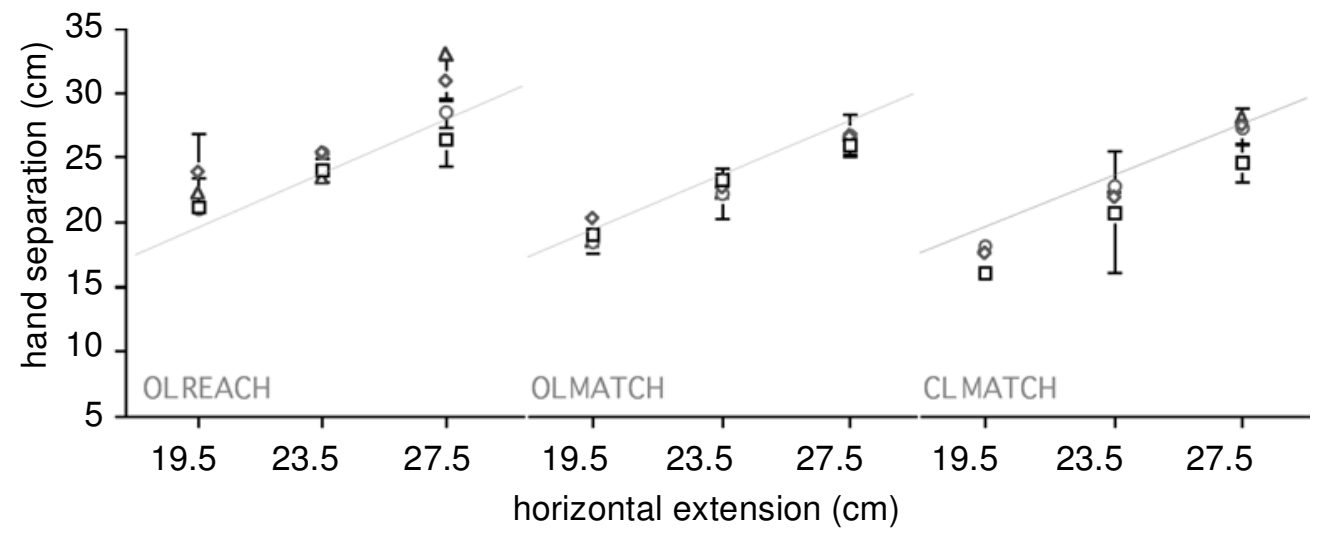

Figure 3. Scaling of bilateral two-hand actions as a function of a surface's horizontal extent. Each graph refers to a different motor response (see the text for a description). Different symbols identify the 4 observers. Each datapoint represents the average of four trials (two repetitions collapsed over two hand starting positions for the actions). Gray lines represent expectations if scaling were perfect. Error bars represent standard errors of the mean, which in most cases were smaller than the plot symbols. OL, open loop; CL, closed loop. 


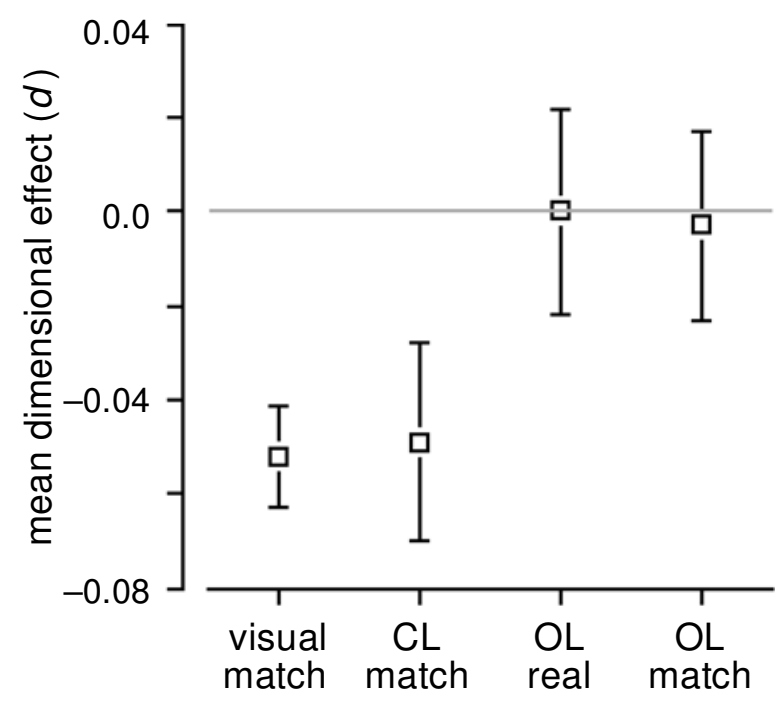

Figure 4. Mean dimensional effects in the four conditions of Experiment 2. Dimensional effects were computed as the difference between the responses on the experimental and the control surfaces, scaled by the width of the control surface. Negative values signify compression; zero values (gray line) signify accurate responses. Plotted points are group averages \pm 1 SEM. CL, closed loop; OL, open loop.

ration after completion of any of the three responses investigated can be taken as a reliable indicator of the spatial representation used in the present motor tasks.

\section{EXPERIMENT 2}

\section{Method}

\section{Observers}

One hundred and twenty members of the Trieste community volunteered. The participants were psychology or achitecture undergraduate and graduate students. All the participants had normal vision or wore prescriptions that were customary for them.

\section{Design and Conditions}

To completely rule out mutual contamination from visual or motor feedback, a fully between design was enforced. The participants were divided into four equal-sized groups, and each group served in a separate experimental condition. Of these, one involved a perceptual discrimination response to estimate a size match (visual judgment task), whereas the other three were identical to the visuomotor responses investigated in Experiment 1. Each participant in any given condition contributed two responses to the experimental surface, which was occluded in the middle by a larger rectangle, as in Figure 1 , left. In addition, each participant also contributed two responses to the control surface, which was identical to the experimental surface but had no occluder. Presentation of the experimental and control surfaces was counterbalanced within conditions.

\section{Materials}

The stimulus materials consisted of rectangular surfaces cut out of light plasterboard and resembled the patterns presented in Figure 1. The experimental and control surfaces that had to be responded to were painted dark gray and were $25 \mathrm{~cm}$ horizontally $\times$ $6 \mathrm{~cm}$ vertically. The occluding surface for the experimental display was painted light gray, and its dimensions were $13 \mathrm{~cm}$ horizontally and $40 \mathrm{~cm}$ vertically. In addition, to collect discrimination responses and estimate a matching size, a set of comparison rectangles was cut that had the same height as the experimental and control surfaces $(6 \mathrm{~cm})$ but a variable length $(19.5-27.5 \mathrm{~cm}$, in steps of $1 \mathrm{~cm})$. In all the conditions, the experimental and control surfaces were attached at the end of a metal rod fixed on a stand. As in Experiment 1, actions were recorded by means of a digital camera (Kodak Dc 240) and were analyzed by importing image files into a personal computer equipped with the Adobe Photoshop image-processing software.

\section{Procedure}

For the three visuomotor responses, the procedures were the same as those used in Experiment 1, with the only difference being that a distance of $57 \mathrm{~cm}$ was used instead of $0.5 \mathrm{~m}$. For the visual judgment task, the exact procedure is detailed below.

Visual judgment. The observers sat in front of the displays at a distance of $57 \mathrm{~cm}$. In each measure, visual size matches were estimated by the method of limits. The experimenter selected one surface from the set of comparisons and held it by hand next to the target surface. The observers indicated which of the compared surfaces appeared larger horizontally. Points of subjective equality (PSE) were estimated by using a full ascending and a full descending series for each of two targets (experimental and control). Thus, each PSE represented the average of two inversion points per condition per observer. Series directions were randomized across observers and targets.

\section{Results and Discussion}

In each experimental condition, measures of dimensional effects were computed as follows. First, for each target and each observer, the average of the two relevant trials was computed. Next, the visually perceived or motorically signaled average extents of the target rectangles were used to compute a dimensional effect index,

$$
d=\frac{O-C}{C}
$$

where $O$ is the visual size match or motoric response for the occluded experimental target and $C$ is the equivalent measure for the control unoccluded target. Note that negative $d$ values signify compression, positive values signify expansion, and zero values signify that the observers were equally accurate in both conditions. Interval estimates of group mean $d$ values in the four conditions are presented in Figure 4 by the condition averages \pm 1 SEM.

In the visual size matches, a compression on the order of $5 \%$ replicated previous studies of Kanizsa's compression illusion (average illusion $=22.8 \mathrm{~cm} \pm 0.2 \mathrm{~cm}$; average control $=24 \mathrm{~cm} \pm 0.1 \mathrm{~cm}$; group mean $d=-0.052$, median $=-0.052$ ). This order of magnitude for $d$ values was representative of most observers, although a couple of observations were weakly in the opposite direction and some showed a much larger compression effect $(S D=$ $0.061)$. The standard error of the mean $(S E M=0.011)$ was almost five times smaller than the observed effect, suggesting that the result is unlikely to have resulted from chance variation. A comparable result was found in closedloop visuomotor matches, which yielded again a compression of the order of $5 \%$ (average illusion $=21.6 \mathrm{~cm}$ $\pm 0.7 \mathrm{~cm}$; average control $=22.8 \mathrm{~cm} \pm 0.5 \mathrm{~cm}$; group mean $d=-0.049$, median $=-0.0505$ ). The larger variance of motor responses produced a larger standard error 
of the mean $d$ in this condition $(S E M=0.021)$. However, the observed effect was still more than two times the size of the error, suggesting that, again, the observed compression is unlikely to have arisen from chance variation.

A different picture emerged from inspecting the two sets of open-loop visuomotor data. In the mimed reaches, the observed dimensional effect was effectively zero (average illusion $=23.4 \mathrm{~cm} \pm 0.8 \mathrm{~cm}$; average control $=$ $23.5 \mathrm{~cm} \pm 0.9 \mathrm{~cm}$; group mean $d=0$, median $=-0.024)$, as it was in the open-loop matches (average illusion = $21.3 \mathrm{~cm} \pm 0.6 \mathrm{~cm}$; average control $=21.5 \mathrm{~cm} \pm 0.7 \mathrm{~cm}$; group mean $d=-0.003$, median $=-0.03$ ). Again, motor responses proved more variable than those provided by participants serving in the perceptual condition. However, standard errors computed for the two-hand reaching and two-hand memory matching conditions were similar in size to that for the closed-loop match $(S E M \mathrm{~s}=0.022$ and 0.02 , respectively). On the basis of this similarity, we excluded the possibility that the null results of the two openloop motor conditions might be attributed to insufficient power.

Thus, interval estimates of dimensional effects indicated that visual matches as well as closed-loop visuomotor matches were indistinguishablefrom an expected value of 5\% for the compression illusion. Conversely, both openloop visuomotor responses were indistiguishable from a $0 \%$ compression. Moreover, $95 \%$ confidence intervals around mean dimensional effects for these responses were close to, but did not cover, the $5 \%$ expectation. These data therefore support the conclusion that open-loop responses were statistically different from the $5 \%$ expectation. To further corroborate this conclusion, we performed separate tests of the open-loop responses against the closed-loop visuomotor matches and the visual matches. The difference between the open-loop visuomotor responses and the visual matches proved statistically reliable $[t(88)=-2.258$, $p<.03$, two-tailed], whereas the difference between the open- and the closed-loop visuomotor responses was marginally significant $[t(88)=-1.87, p<.065$, two-tailed $]$.

As in the first experiment, to check for interactions with starting positions, data in all three visuomotor conditions were subjected to an additional analysis after separating the measures. The outcome of this analysis is summarized graphically in Figure 5. In the closed-loop matches, interhand distances were larger with the control than with the experimental surface, independent of starting position. In the two open-loop responses, interhand distances were strongly affected by the starting position for the action but were similar with both surfaces.

\section{GENERAL DISCUSSION}

We report a new functional dissociation between visual judgments and visually directed actions in healthy observers. When a horizontally elongated surface is occluded in the middle by a larger surface, it appears narrower than its true width. Our data indicate that a similar compression effect occurs for closed-loop visuomotor matches of size, but not for otherwise comparable open-loop "mimed" reaching or size-matching visuomotor responses. Given that all these motor responses were produced while exactly the same displays were observed, the present results are immune from the criticism of inadequate matching of perceptual and motor conditions (V. H. Franz et al., 2000).

The present results are generally consistent with the possibility of functionally distinct visual streams for vi-

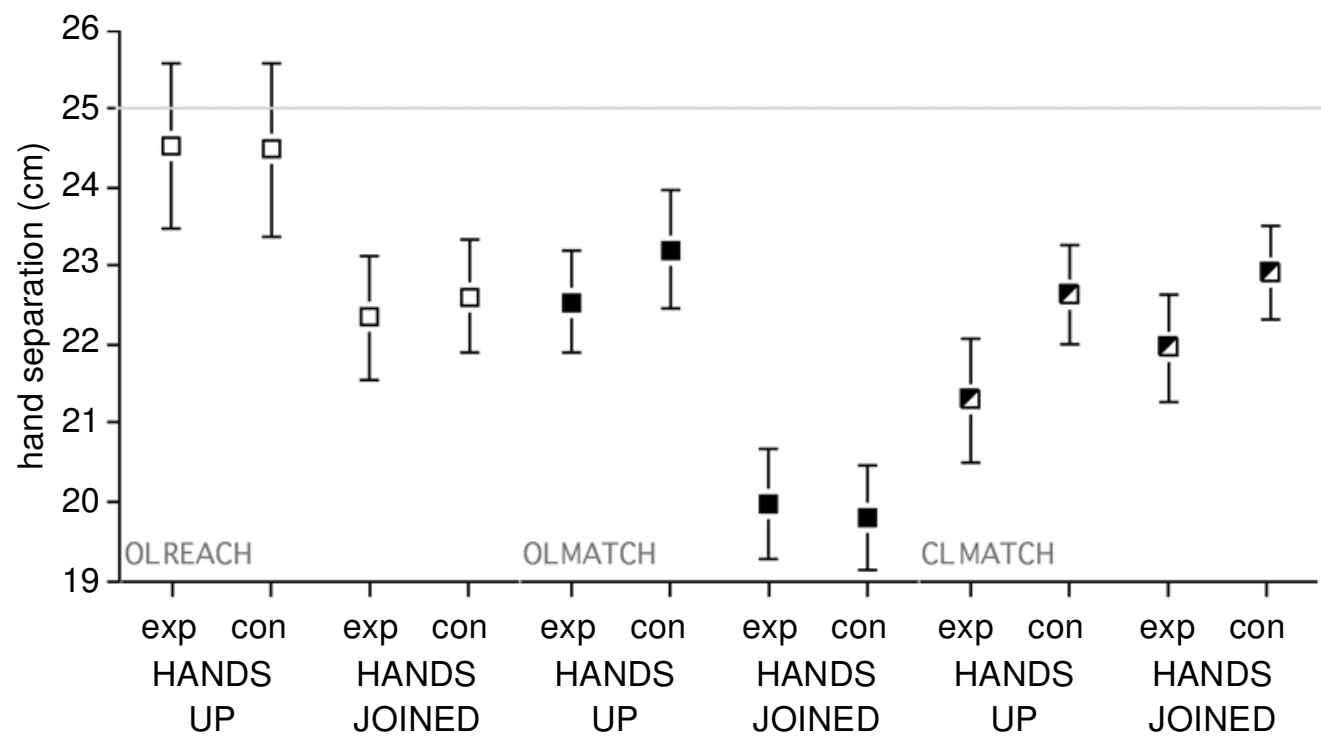

Figure 5. Effects of hand starting position in Experiment 2. Plotted data are group averages $\pm 1 S E M$. The gray horizontal line marks the actual surface width. OL, open loop; CL, closed loop; exp, experimental; con, control. 
sual awareness and visually guided actions. In accord with this general idea, closed-loop visuomotor matches produced while the stimulus was continuously available for the on-line control of the matching gesture were similar to purely visual PSEs as measured by a discrimination procedure. Conversely, open-loop visuomotor matches produced on the sole basis of an initial motor program and executed without further feedback proved immune to the illusory compression, as one would expect if these used a spatial map that was not influenced by the occluding surface. Contrary to the current characterization of the TVSH, however, we found immunity from the compression illusion even in the open-loop visuomotor matching responses. In an investigation of dimensional effects in size-contrast illusions, Haffenden and Goodale (1998) asked participants to match target sizes with the aperture of the index finger and the thumb. They found such manual estimates to be strongly influenced by size contrast, whereas finger apertures when the object was actually picked up were not. Although they asked observers to perform the match without seeing their hands or the targets, Haffenden and Goodale suggested that their manual estimation task was influenced by ventral stream processes.

Given the similarity of our open-loop visuomotor matches to Haffenden and Goodale's (1998) motor estimation task, one would have expected that our visumotor matches would have shown compression, but they did not. Several explanations may account for the difference. First of all, we note that criteria for classifying tasks as dorsal or ventral independently of experimental outcome are not always clear-cut (Carey, 2001). For instance, it could be that our open-loop "match" effectively isolated the initial scaling of hand separation while preparing for a complete bilateral grasp. Alternatively, the difference may reflect the possibility of using body-centered coordinates for hand position in our open-loop matching task, which may be harder to do when size estimations are provided for smaller surfaces, using the thumb and index fingers. Further work will be needed to test these possibilities.

Besides providing evidence for a perception-action dissociation in personal space, our results provide indications suggesting some general biases in visually planned actions. When providing motoric responses to the perceived width of the presented surfaces, our observers showed a general underestimation bias. This bias is consistent with well-known visual angle effects in size constancy tasks (see, for instance, McKee \& Welch, 1992). Interestingly, however, this bias was small when they reached for the surface and more substantial when they motorically matched its width by memory or by looking. This finding suggests that size constancy tasks might provide an interesting domain (and possibly one more general than illusion patterns) for further investigations of perception-action dissociations.

In addition, our results also suggest a strong motoric bias from the starting position of the reaching or matching action. In reaching as well as in memory matching (our two open-loop conditions), the observers underestimated substantially more when they started with their hands joined in front of the chest than when they started with their hands above the shoulders. This result is surprising in that it runs counter to the idea that different actions would tend to equalize the amount of space traveled. The joined hands were very close to the sides of the target surface, and yet the participants consistently tended to stop their actions shorter than they did with the much farther raised hands. Such asymmetries in a visuomotor size constancy task are interesting, in that they may offer a way to study the involvement of different observer-centered spatial maps for the control of action. We note that such a bias was exhibited by the data in our second experiment, which used a fully between design, but not in our first experiment, in which each participant performed many more actions. This difference may reflect some form of motor learning based on proprioceptive and visual information in the closed-loop condition.

\section{REFERENCES}

Aglioti, S., DeSouza, J. F., \& Goodale, M. A. (1995). Size-contrast illusions deceive the eye but not the hand. Current Biology, 5, 679685.

Bhalla, M., \& Proffitt, D. R. (1999). Visual-motor recalibration in geographical slant perception. Journal of Experimental Psychology: Human Perception \& Performance, 25, 1076-1096.

Boessenkool, J. J., NiJHof, E. J., \& ERKelens, C. J. (1999). Variability and correlations of bi-manual pointing movements. Human Movement Science, 18, 525-552.

Brenner, E., \& SMeEts, J. B. (1996). Size illusion influences how we lift but not how we grasp an object. Experimental Brain Research, 111, 473-476.

BRIDGEMAN, B. (1999). Separate representation of visual space for perception and visually guided behavior. In G. Aschersleben \& T. Bachmann (Eds.), Cognitive contributions to the perception of spatial and temporal events (Advances in psychology, Vol. 129, pp. 3-13). Amsterdam: North-Holland/Elsevier.

Bridgeman, B., Kirch, M., \& Sperling, A. (1981). Segregation of cognitive and motor aspects of visual function using induced motion. Perception \& Psychophysics, 29, 336-342.

Bridgeman, B., Lewis, S., Heit, G., \& Nagle, M. (1979). Relationship between cognitive and motor systems of visual position perception. Journal of Experimental Psychology: Human Perception \& Performance, 5, 692-700.

Bruce, V., Green, P. R., \& Georgeson, M. A. (1996). Visual perception: Physiology, psychology, and ecology (3rd ed.). Hove, U.K.: Psychology Press.

BRuno, N. (2001). When does action resist visual illusions? Trends in Cognitive Sciences, 5, 379-382.

CAREY, D. P. (2001). Do action systems resist visual illusions? Trends in Cognitive Sciences, 5, 109-113.

Cutting, J. E., \& Vishton, P. M. (1995). Perceiving layout and knowing distances: The integration, relative potency, and contextual use of different information about depth. In W. Epstein \& S. Rogers (Eds.), Handbook of perception and cognition: Vol. 5. Perception of space and motion (pp. 69-117). San Diego: Academic Press.

Daprati, E., \& Gentilucci, M. (1997). Grasping an illusion. Neuropsychologia, 35, 1577-1582.

Delucia, P. R., Tresilian, J. R., \& Meyer, L. E. (2000). Geometrical illusions can affect time-to-contact estimation and mimed prehension. Journal of Experimental Psychology: Human Perception \& Performance, 26, 522-567.

Franz, E. A., Zelaznik, H. N., Swinnen, S., \& Walter, C. (2001). Spatial conceptual influences on the coordination of bimanual actions: When a dual task becomes a single task. Journal of Motor Behavior, 33, 103-112. 
Franz, V. H., Gegenfurtner, K. R., Bülthoff, H. H., \& Fahle, M. (2000). Grasping visual illusions: No evidence for a dissociation between perception and action. Psychological Science, 11, 20-25.

Gentilucci, M., Chieffi, S., Daprati, E., Saetti, M. C., \& Toni, I. (1996). Visual illusion and action. Neuropsychologia, 34, 369-376.

GERBINO, W. (1979). Il ruolo della forma nelle modificazioni di grandezza dovute a completamento amodale [The role of form in size modifications due to amodal completion]. Reports from the Institute of Psychology, University of Trieste.

Haffenden, A. M., \& Goodale, M. A. (1998). The effect of pictorial illusion on prehension and perception. Journal of Cognitive Neuroscience, 10, 122-136.

Haffenden, A. M., Schiff, K. C., \& Goodale, M. A. (2001). The dissociation between perception and action in the Ebbinghaus illusion: Nonillusory effects of pictorial cues on grasp. Current Biology, 11, 177-181.

JACKsOn, S. R., \& SHAW, A. (2000). The Ponzo illusion affects grip-force but not grip-aperture scaling during prehension movements. Journal of Experimental Psychology: Human Perception \& Performance, 26, 418-423.

KAnIZSA, G. (1972). Schrumpfung von visuellen Feldern bei amodaler Ergänzung [Shrinkage of the visual field due to amodal completion]. Studia Psychologica, 14, 208-210.

Kanizsa, G. (1975). Amodal completion and phenomenal shrinkage of surfaces in the visual field. Italian Journal of Psychology, 2, 187-195.

Loomis, J. M., Da Silva, J. A., Fujita, N., \& Fukushima, S. S. (1992). Visual space perception and visually directed action. Journal of Experimental Psychology: Human Perception \& Performance, 18, 906921.

Luccio, R. (1983). Effetti dimensionali del completamento amodale [Dimensional effects of amodal completion]. Ricerche di Psicologia, 7, 119-136.

Marteniuk, R. G., Leavitt, J. L., MacKenzie, C. L., \& Athenes, S. (1990). Functional relationship between grasp and transport components in a prehension task. Human Movemement Science, 9, 149-176.

McKeE, S. P., \& Welch, L. (1992). The precision of size constancy. Vision Research, 32, 1447-1460.

Milner, A. D., \& Goodale, M. A (1995). The visual brain in action. Oxford: Oxford University Press.

Milner, A. D., Perrett, D. I., Johnston, R. S., Benson, P. J., Jordan, T. R., \& HeELEY, D. W. (1991). Perception and action in "visual form agnosia." Brain, 114, 405-428.
Otto-de-Haart, E. G., Carey, D. P., \& Milne, B. (1999). More thoughts on perceiving and grasping the Müller-Lyer illusion. Neuropsychologia, 37, 1437-1444.

Pavani, F., Boscagli, I., Benvenuti, F., Rabuffetti, M., \& Farne, A. (1999). Are perception and action affected differently by the Titchener circles illusion? Experimental Brain Research, 127, 95-101.

Perenin, M., \& Vighetto, A. (1983). Optic ataxia: A specific disorder in visuomotor coordination. In A. Hein \& M. Jeannerod (Eds.), Spatially oriented behavior (pp. 305-326). New York: Springer-Verlag.

Plodowsky, A., \& JACKson, S. R. (2001). Getting to grips with the Ebbinghaus illusion. Current Biology, 11, 304-306.

Post, R. B., \& WELCH, R. B. (1996). Is there dissociation of perceptual and motor responses to figural illusions? Perception, 25, 569-581.

Proffitt, D. R., Bhalla, M., Gossweiler, R., \& Midgett, J. (1995). Perceiving geographical slant. Psychonomic Bulletin \& Review, 2, 409428.

TAMPIERI, G. (1979). The shrinkage of amodally completed surfaces in figure-ground situations. Italian Journal of Psychology, 6, 53-57.

VeZZANI, S. (1999). Shrinkage and expansion by amodal completion: A critical review. Perception, 28, 935-947.

Vishton, P. M., \& CutTing, J. E. (1995). Veridical size perception for action: Reaching vs. estimating [Abstract]. Investigative Ophthalmology \& Visual Science, 36, S358.

Vishton, P. M., Cutting, J. E., \& Rea, J. G. (1997). Titchener circles and horizontal-vertical illusions do not affect manual prehension or judgements of absolute size: Müller-Lyer illusion affects both [Abstract]. Investigative Ophthalmology \& Visual Science, 36, S643.

Vishton, P. M., Rea, J. G., Cutting J. E., \& Nunez, L. N. (1999). Comparing effects of the horizontal-vertical illusion on grip scaling and judgment: Relative vs. absolute, not perception vs. action. Journal of Experimental Psychology: Human Perception \& Performance, 25, 1659-1672.

Wraga, M., Creem, S. H., \& Proffitt, D. R. (2000). Perception-action dissociations of a walkable Müller-Lyer configuration. Psychological Science, 11, 239-243.

ZANUTTINI, L. (1977). Amodal shrinkage during condition of illusory dilatation of surfaces. Italian Journal of Psychology, 4, 101-111.

(Manuscript received December 14, 2000; revision accepted for publication October 19, 2001.) 